\title{
Prospecção fitoquímica de Himatanthus drasticus Plumel (Apocynaceae), da mesorregião leste maranhense
}

\begin{abstract}
LUZ, H.S." SANTOS, A.C.G.2; LIMA; F.C.3; MACHADO; K.R.G. ${ }^{4}$
1Programa de Pós-Graduação em Ciência Animal, Universidade Estadual do Maranhão, Centro de Ciências Agrárias, Cidade Universitária Paulo VI. C.P. 09. Tirirical - CEP. 65055-970 - São Luís/MA. Brasil. hildecy.luz@ hotmail.com; '2Programa de Pós-Graduação em Ciência Animal, Universidade Estadual do Maranhão, Centro de Ciências Agrárias, Cidade Universitária Paulo VI. C.P. 09. Tirirical - CEP. 65055-970 - São Luís/MA. Brasil. santos.clara @ig.com.br; ${ }^{3}$ Curso de Zootecnia, Universidade Estadual do Maranhão, Centro de Ciências Agrárias, Centro de Ciências Agrárias, Cidade Universitária Paulo VI. C.P. 09. Tirirical - CEP. 65055-970 - São Luís/MA. Brasil.fcarneiro.vet11@yahoo.com.br; "4Química, Universidade Federal do Maranhão, Av. dos Portugueses, Campus do Bacanga, slnº- CEP 65.080-040 São Luís-MA, Brasil. kedmarejane@gmail.com
\end{abstract}

RESUMO: O uso indiscriminado de Himatanthus drasticus (Janaúba) por pequenos caprinocultores no controle de verminose em pequenos ruminantes foi o que motivou a realização do estudo fitoquímico do mesmo. Cascas da janaúba foram coletadas na mesorregião Leste do Maranhão e conduzidas aos laboratórios de Nutrição da Universidade Estadual do Maranhão e de Produtos Naturais da Universidade Federal do Maranhão para a identificação botânica e fitoquímica pela metodologia da Prospecção Preliminar e CCD, realizando testes para as diversas classes de metabólitos secundários. A partir das cascas do vegetal moído e desidratado foi realizado o preparo do extrato bruto (EB). O material foi colocado em uma mistura hidroalcoólica de EtOH: $\mathrm{H}_{2} \mathrm{O}(7: 3 \mathrm{v}: \mathrm{v})$, e submetido a agitação mecânica esporádica. Os Subextratos foram obtidos a partir do EBHA pelo processo de partição líquido-líquido, ETOH: $\mathrm{H}_{2} \mathrm{O}(2: 1, \mathrm{v}: \mathrm{v})$. As misturas foram preparadas com os seguintes solventes orgânicos de polaridades crescentes: hexano, acetato de etila e butanol. As análises cromatográficas evidenciaram a presença de grupos de metabólitos secundários no extrato e nos subextratos. As classes de metabólitos secundários que apresentaram maior expressividade na análise de prospecção foram os alcaloides e taninos, enquanto que na analise por CCD foram os flavonoides e terpenos, indicando o potencial da ação farmacológica das cascas de H. drasticus.

Palavras-chave: Bioatividade, Fitoterápico, Maranhão.

ABSTRACT: Phytochemical screening of Himatanthus drasticus Plumel (Apocynaceae), from the eastern mesoregion of Maranhão, Brazil. The indiscriminate use of the plant Himatanthus drasticus (Janaúba) by small goat farmers to control nematode parasites in small ruminants was the aim of this phytochemical study of the plant. Barks were collected in the eastern mesoregion of the state of Maranhão, Brazil, and sent to the laboratory for botanical and phytochemical identification by the methodology of Preliminary Prospecting and TLC, testing for the various classes of secondary metabolites. From the ground, dehydrated bark, we conducted the preparation of the crude extract (CE). The material was placed in a water-alcohol mixture of EtOH:H2O (7:3 v:v) and subjected to sporadic mechanical agitation. The subextracts were obtained using the HACE process by liquid-liquid partition, ETOH:H2O (2:1, v:v). The mixtures were prepared with organic solvents of increasing polarity, such as hexane, ethyl acetate and butanol. The GC analysis showed the presence of groups of secondary metabolites in the extract and subextractes. The classes of secondary metabolites that showed greater expressiveness are the alkaloids and tannins, when analyzed by prospecting, and flavonoids and terpenes, when analyzed by TLC, and $H$. drasticus presents elements indicative of pharmacological action.

Keywords: Bioactivity, Phytotherapic, Maranhão.

Recebido para publicação em 05/09/2012

Aceito para publicação em 23/04/2014

$10.1590 / 1983-084 X / 12 \_114$

Rev. Bras. PI. Med., Campinas, v.16, n.3, supl. I, p.657-662, 2014. 


\section{INTRODUÇÃO}

A utilização de plantas medicinais como alternativa terapêutica vem atingindo um público cada vez maior e este crescimento requer dos pesquisadores um maior empenho, no sentido de fornecer informações relativas à correta identificação, do sistema produtivo destas plantas e preparo adequado de medicamentos.

A família Apocynaceae Jussaeu (1789) pode ser considerada uma das maiores fontes vegetais de constituintes químicos de utilidade na medicina moderna. Várias substâncias têm sido isoladas a partir de espécies do táxon. Muitas delas representam protótipos de classes farmacológicas distintas de fármacos e fazem parte da história da farmacologia e da terapêutica. $O$ isolamento de alcalóides de plantas pertencentes aos gêneros Rauvolfia, Vinca, Catharanthus e Alstonia são exemplos que podem ser citados Di Stasi \& Hiruma-Lima (2002). Na literatura não há relatos para a presença deste composto para $H$. sucuuba. Entretanto, são comprovadas as atividades gastroprotetivas para os alcalóides indólicos de $\mathrm{H}$. lancifolius (Baggio et al., 2005).

A família Apocynaceae compreende aproximadamente 250 gêneros presentes em duas subfamílias, Apocynoideae e Plumerioideae e 2000 espécies, distribuídas pelas regiões tropicais e subtropicais do planeta. São plantas de hábito variado, ervas, subarbustos, árvores e trepadeiras encontradas na flora brasileira e, particularmente, no Rio de Janeiro; segundo levantamento realizado por Novaes \& Rapoporte (1996) existindo cerca de 80 espécies.

Himatanthus drasticus é conhecido popularmente como tiborna, jasmim-manga e raivosa em Minas Gerais e Bahia, janaúba no Ceará, pau-de-leite no Piauí, janaguba no Rio Grande do Norte, sucuúba na Amazônia (Plumel, 1991).

O látex de $H$. drasticus tem indicação popular contra o câncer de pulmão e linfático, vermes intestinais, febre e úlceras gástricas (Lorenzi \& Matos, 2008). Estudos realizados por Mesquita et. al., (2005) nas raízes de $H$. drasticus indicaram que apresentam efeito leishmanicida contra promastigotas de Leishmania donovani. Estudos prévios com as espécies de Himatanthus demonstraram atividade farmacológica contra o carcinoma epidermóide nasofaringe de humano (Perdue \& Blomster, 1978); antiinflamatória e analgésica (Miranda et al., 2000); antimicrobiana (Souza et al., 2004) e efeito gastroprotetor (Baggio et al., 2005).

Himatanthus drasticus, como outras espécies do gênero Himatanthus foram escassamente relatadas em relação aos seus estudos fitoquímicos e suas atividades biológicas. Colares et al., (2008a) isolaram e identificaram do extrato etanólico da casca o triterpeno lupeolcinamato que provavelmente é uma substância com perspectivas de apresentar atividade antitumoral. Outro trabalho com ação farmacológica dessa espécie foi o efeito gastroprotetor do látex através de lesão gástrica induzida por etanol e indometacina (Colares et al., 2008b). Já da espécie $H$. sucuuba foi isolado o iridóide fulvoplumierina que apresentou atividade contra carcinoma nasofaríngeo epidermóide humano (Perdue \& Blomster, 1978).

A análise fitoquímica se faz necessária para conhecimento dos componentes químicos encontrados nos vegetais, para se chegar ao isolamento de princípios ativos importantes na produção de novos fitoterápicos. O método cromatográfico destaca-se entre os diversos métodos de análise como um dos mais frutíferos em resultados satisfatórios e mais rico em número de técnicas. Como processo de análise imediata tem permitido o fracionamento de misturas em seus componentes com maior precisão e com menor consumo de tempo e trabalho, à medida que vêm sendo desenvolvidos novos materiais e novas técnicas (Matos 1997).

O uso do vegetal como medicinal pela população, a utilização na alimentação de Himantanthus drasticus em animais de produção como controle de verminose e o fato de não haver estudos sobre a atividade anti-helmíntica com a espécie do gênero foi o que motivou a realização da análise química para rastrear os possíveis grupos de compostos relacionados com a bioatividade dos extratos.

\section{MATERIAL E MÉTODOS}

\section{Material botânico}

A coleta de $H$. drasticus foi realizada nas áreas florestais do município de Caxias, MA. Localizado nas coordenadas (latitude $4^{\circ} 51^{\prime} 32^{\prime \prime} \mathrm{S}$ e longitude $43^{\circ} 21^{\prime} 22^{\prime \prime}$ O), altitude de $66 \mathrm{~m}$, em uma área de cerrado, na mesorregião Leste Maranhense (IBGE, 2008).

A identificação e classificação botânica do vegetal $H$. drasticus foi realizada através de chaves analíticas, comparações com as descrições e ilustrações da literatura especializada, segundo a classificação padronizada por Spina (2004) e Lorenzi (1992), utilizando-se folhas, flores, frutos e sementes, sendo realizada no Laboratório de Botânica, Herbário Ático Seabra, da Universidade Federal do Maranhão (UFMA), onde a exsicata se encontra catalogada e depositada sob o registro $n^{\circ}$ 01032. 


\section{Preparo do extrato}

A partir das cascas vegetal moído, desidratado e pesada $(3 \mathrm{~kg})$ foi realizado o preparo do extrato bruto (EB). O material foi colocado sob maceração a frio em uma mistura hidroalcoólica de EtOH: $\mathrm{H}_{2} \mathrm{O}$ (7:3 v:v) e submetido a agitação mecânica esporádica, empregando-se o método de Soxleht, seguida da purificação do extrato em coluna cromatográfica de silica gel $(1: 0,5)$, obtendo-se o extrato bruto hidroalcoólico da janaúba (EBHA-janaúba). Realizou-se misturas de solventes orgânicos de polaridades crescentes, como o hexano (S.He.), acetato de etila (S.Ac.), butanol (S.Bu.) e aquoso (S.Aq.), subextratos esses aplicados em Coluna Cromatográfica CC) e Cromatografia em Camada Delgada (CCD), seguindo-se os métodos padronizados por Matos (1999), Moreira (1979) e modificada por Nakashima (1993).

\section{RESULTADOS E DISCUSSÃO}

\section{Fitoquímica}

O extrato hidroalcóolico obtido das cascas de $H$. drasticus foi analisado para se conhecer o perfil de seus metabólitos secundários, de acordo com metodologia descrita por Matos, (1999). A análise cromatográfica evidenciou a presença de alcalóide no extrato bruto (Tabela 1). Esse resultado vem corroborar com estudos já realizados anteriormente com o gênero Himatanthus através da quimiotaxia, revelando principalmente a presença de alcalóides e outros metabólitos na espécie da familia Apocynaceae (Kisakurek \& Hesse (1980); Grupo (1998); Kazmi (1989), Franca \& Brown et al. (2000); Massiot \& Boumendel (1992).

Os resultados obtidos da triagem fitoquímica do EBHA e dos subextratos foi positiva para taninos, com exceção do S.He. Os taninos são usados tradicionalmente da diarréia, hipertensão, reumatismo, hemorragias e em processos inflamatórios, possuindo atividades comprovadas tais como: bactericida, fungicida, antiviral, moluscicida e antitumoral (Simões et al., 2007). E, ausência de heterosídeos flavônicos e esteróides para o EBHA-Janaúba. No S.Bu estavam presente com maior intensidade as saponinas e triperternos; enquanto, S.Aq. apresentou somente as saponinas. Os esteróides só foram encontrado no S.He. (Tabela 1).

Os flavonóides encontrados nos subextratos do $H$. drasticus (Janaúba) apresentam características químicas e biossintéticas já relatadas por Ângelo \& Jorge 2007.

Nesse estudo foi verificada apenas a presença de triterpenos no EBHA-Janaúba e no S.Bu (Tabela 1). Os terpenos estão envolvidos em diferentes funções nos vegetais, desde a composição de alguns óleos essenciais de plantas, o que confere características como a atração de polinizadores; ação inseticida e antimicrobiana (sesquiterpenos), dentre outras (Oliveira, 2007).

Comparando os resultados desta pesquisa com as análises fitoquímicas, realizadas através da técnica de CCD com Himatanthus obovatus coletadas no cerrado do município de Caxias, MAe Timon, MA foi observada a presença de esteróides, flavonóides, alcalóides, taninos e saponinas (Santos, 2002). Já Miranda et al. (2000) observaram frações hexânicas, triterpenos e iridóides nos extratos hidroalcoólicos obtidos das cascas e folhas de $H$. sucuuba.

Honda, (1990) no estudo de plantas em Mato Grosso do Sul realizou triagem fitoquímica de 100 espécies vegetais daquela região, dentre essas o gênero Himatanthus, utilizando a Técnica de Prospecção de constituintes químicos de extratos de plantas, não obtendo resultados positivos para a classe de metabólitos secundários de alcaloides.

O perfil cromatográfico em CCD foi realizado a partir do EBHA-Janaúba para os subextratos de S.He., S.Ac., S.Bu. e S.Aq. observando-se que a fase móvel utilizada foi eficiente apenas na separação das substâncias componentes do S.He. No entanto, para a avaliação cromatográfica do EBHA e os demais subextratos foi necessário aumentar a polaridade da fase móvel de modo a promover uma melhor interação com as substâncias, permitindo assim o arraste delas ao longo da placa (Figura 1).

$\mathrm{Na}$ fase móvel, com o uso dos mesmos solventes, houve aumento de $\mathrm{MeOH}$ e consequentemente de sua polaridade. Foi observado, então, que é possível se obter uma melhor separação entre os compostos presentes em cada amostra estudada.

No cromatograma foi observada maior concentração relativa de componentes mais polares do que dos menos polares, notando-se que no S.Aq. e no S.Bu. o perfil cromatográfico é semelhante entre eles e também com a metade inferior do EBHA.

No S.Ac. ocorreu menor quantidade de "manchas", o que é indicativo de pouca quantidade de substâncias componentes nessa fração, o que a torna uma fração de interesse a ser fracionada na procura de frações mais limpas, ou mesmo, até de substâncias isoladas. Tomando como base essa avaliação cromatográfica preliminar foi possível escolher o melhor eluente para o fracionamento do S.Ac. por CC (Figura 2).

Os S.Bu., S.Ac. e S.Aq. apresentaram flavonóis, o S.Ac também apresentou flavanonas, enquanto o S.Aq. apresentou catequinas. O S.He. apresentou resultado positivo para flavonas, flavonóis

Rev. Bras. PI. Med., Campinas, v.16, n.3, supl. I, p.657-662, 2014. 
e xantonas. Do ponto de vista farmacológico, esses compostos possuem atividade anti-séptica, antiinflamatória e podem inibir atividade enzimática (Bruneton, 1995).

Os S.Ac., S.Bu. e S.Aq. apresentaram resultados positivos para presença de saponinas. Estudos comprovam a ação das saponinas nas atividades antiinflamatórias, antihelminticas e antivirais. Para saponinas os testes foram considerados positivos. As saponinas, também chamadas saponosídeos, formam um grupo particular de heterosídeos derivados dos triterpenos tetracíclicos. Elas reduzem a tensão superficial da água, e causam, in vitro, a hemólise de eritrócitos. As saponinas, apesar de muito usadas na indústria farmacêutica, apresentam propriedades tóxicas aos seres humanos (Vickery \& Vickery, 1981). Sua ação lipofílica facilita a complexação das saponinas com esteróides, proteínas e fosfolipídeos das membranas celulares alterando a permeabilidade das mesmas, ou causando sua destruição (Schenkel et al., 2001).

Os esteróides e triterpenos apresentaram resultados positivos nos S.He. e S.Bu. Essa classe de metabólitos secundários tem maior afinidade, principalmente pelos solventes como o n-hexano e o n-butanol, sendo estes utilizados no presente estudo e corroboram com os descritos nas frações hexânicas do látex de $H$. sucuuba por Miranda et al. (2000) e das cascas do caule por Silva et al. (1998a).

Os metabólitos secundários cumarinas apresentaram resultado positivo para o EBHAjanaúba.

Nos cromatogramas de CCD foi observado que algumas frações apresentaram "manchas" com o mesmo deslocamento, sendo possível reuní-las para obter frações semelhantes com maior massa. Foi necessário reduzir a quantidade de $\mathrm{MeOH}$ e aumentar a de $\mathrm{CHCl}_{3}$ (Figura 3) para diminuir a polaridade da fase móvel utilizada.

A polaridade de algumas frações da fase móvel foi alta, ocorrendo o arraste das substâncias até o topo, havendo necessidade de diminuir a polaridade da fase móvel reduzindo a quantidade de $\mathrm{MeOH}$ e aumentando a de $\mathrm{CHCl}_{3}$.

Analisando-se o cromatograma foi possível observar que, com a diminuição da polaridade da fase móvel houve uma melhor separação das substâncias presentes nas frações, sendo que a fração FR8 apresentou um perfil cromatográfico interessante devido à presença de "manchas" com maior intensidade (mais concentradas), e um melhor deslocamento (melhor resolução) ao longo da placa (Figura 4).

Com esses resultados, pode-se concluir que a triagem fitoquímica do vegetal $H$. drasticus demonstra a presença de metabólitos secundários expressivos, tais como alcalóides, taninos, flavonóides, fenóis e triperpenóides, substâncias químicas com potencial farmacológico. Registrando pela primeira vez o estudo fitoquímico desse vegetal.

\section{CONCLUSÃO}

As análises fitoquímicas realizadas revelaram que Himatanthus drasticus apresenta compostos pertencentes às classes dos alcalóides e taninos, que podem ser potencialmente ativos em modelos biológicos e farmacológicos. Também

TABELA 1. Prospecção química do EBHA de Himatanthus drasticus (Plumel) e dos Subextratos.

\begin{tabular}{|c|c|c|c|c|c|}
\hline \multirow{2}{*}{$\begin{array}{l}\text { Metabólitos } \\
\text { Secundários }\end{array}$} & \multirow{2}{*}{$\begin{array}{c}\text { EBHA-Ja- } \\
\text { naúba }\end{array}$} & \multicolumn{4}{|c|}{ Subextratos } \\
\hline & & S. $\mathrm{He}$ & S. Ac & S. Bu & S. Aq \\
\hline Taninos & +++ & - & ++ & ++ & ++ \\
\hline $\begin{array}{l}\text { Heterosídeos Fla- } \\
\text { vônicos }\end{array}$ & - & ++ & ++ & ++ & ++ \\
\hline Alcalóides & +++ & - & - & - & - \\
\hline Saponinas & +++ & - & + & +++ & +++ \\
\hline Triterpenos & +++ & - & - & +++ & - \\
\hline Esteróides & - & +++ & - & - & - \\
\hline Cumarinas & +++ & NR & NR & NR & NR \\
\hline \multicolumn{2}{|c|}{$\begin{array}{l}\text { +: reação fracamente positiva } \\
++: \text { reação positiva } \\
+++ \text { : reação fortemente positiva } \\
\text { : ausente NR: não realiz }\end{array}$} & \multicolumn{4}{|c|}{$\begin{array}{l}\text { EBHA-janaúba= estrato bruto hidroalcoolico janaúba } \\
\text { S.He= subextrato hexânico } \\
\text { S. Ac. =subextrato Acetado de Etila } \\
\text { S.Bu= subextrato n-butanólico } \\
\text { S. Aq. = subextrato Aquoso }\end{array}$} \\
\hline
\end{tabular}

Rev. Bras. PI. Med., Campinas, v.16, n.3, supl. I, p.657-662, 2014. 


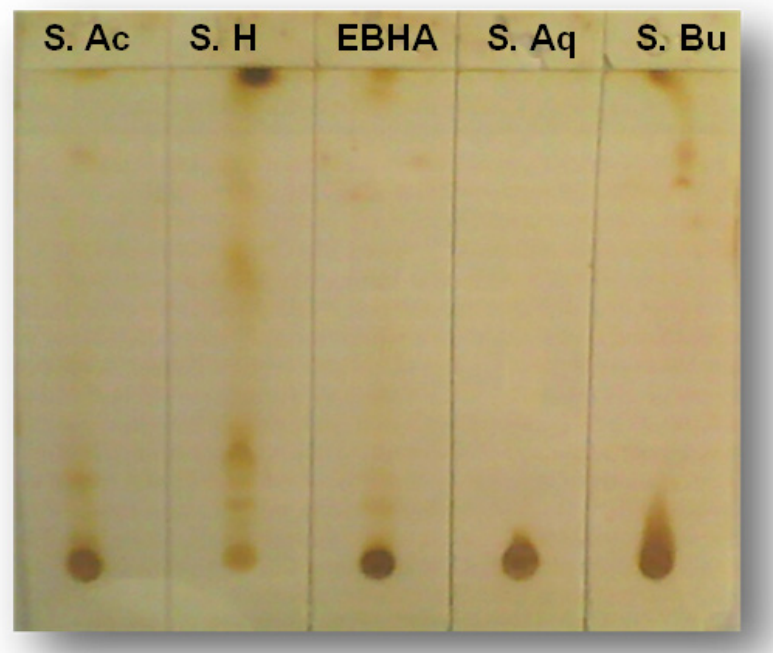

FIGURA1. Cromatogramas do EBHAde Himatanthus drasticus e Subextratos. Fase móvel: $\mathrm{CHCl}$ : $\mathrm{MeOH}$ $(8,5: 1,5)$ (v:v). Revelação com vapores de iodo.

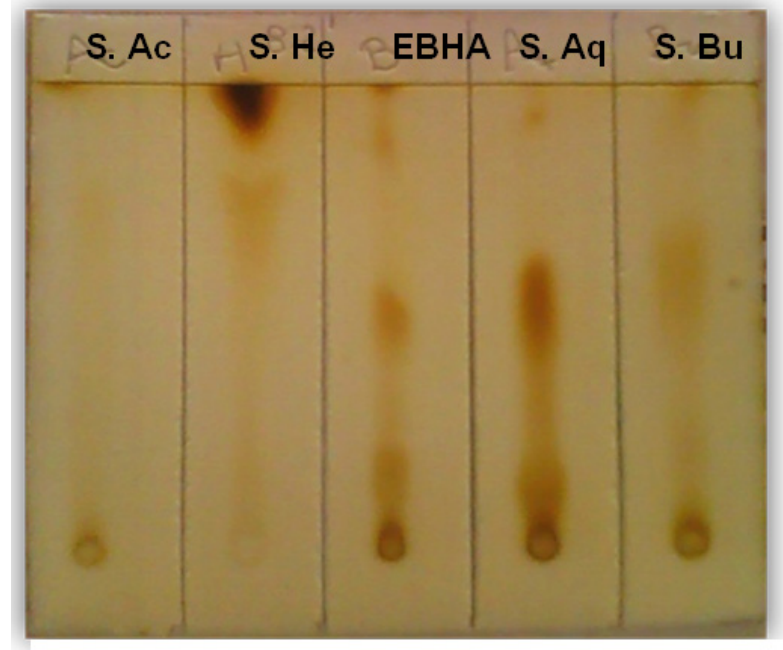

FIGURA2. Cromatogramas do EBHAde Himatanthus drasticus e Subextratos. Fase móvel: $\mathrm{CHCl}$ : $\mathrm{MeOH}$ (8,0:2,0 v:v). Revelação com vapores de iodo.

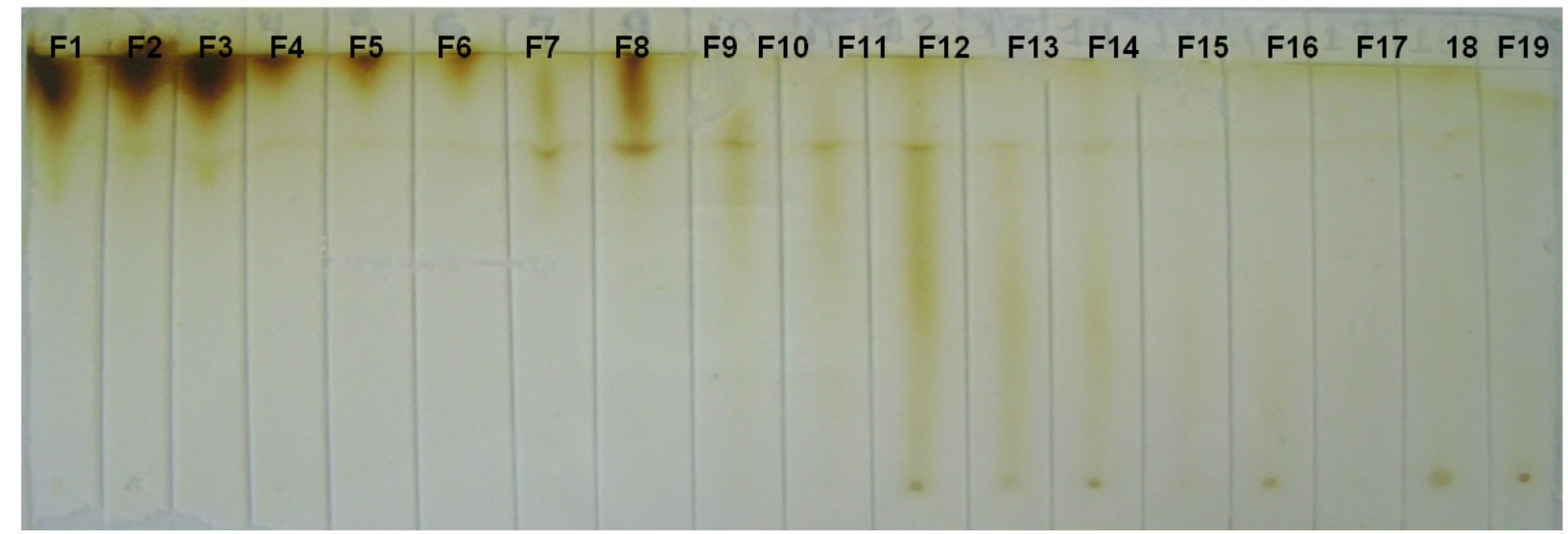

FIGURA 3. Cromatogramas das frações 1-19 obtidas pelo fracionamento da Subextrato Acetato. Fase móvel: $\mathrm{CHCl}_{3}$ : eOMH (7,5:2,5 v:v). Revelação com vapores de iodo.

\begin{tabular}{|l|l|l|l|l|l|}
\hline FR1 & FR4 & F7 & FR8 & FR10 & FR15 \\
\hline & & & & \\
& & & & \\
& & & & \\
& & & & \\
& & & & \\
\hline
\end{tabular}

FIGURA 4. Cromatogramas das frações reunidas pelo fracionamento do Subextrato Acetato. Fase móvel: $\mathrm{CHCl}_{3}: \mathrm{MeOH}$ (8,5:1,5 v:v). Revelação com vapores de iodo.

apresenta cumarinas com potencial antimicrobiano, antinflamatório, antiviral e antioxidante; além do potencial de ação antifúngica e hipocolesterolemiante das saponinas.

\section{AGRADECIMENTOS}

À Universidade Estadual do Maranhão (UEMA), ao Programa de Pós Graduação em Ciência Animal e a Universidade Federal do Maranhão (UFMA). Ao Prof. Dr. Roberto Sigfrido 
Gallegos Olea (UFMA) que permitiu que todo o trabalho de fitoquímica fosse realizado no Laboratório de Produtos Naturais (LPN). Ao Prof. Dr. Antônio Carlos Romão Borges (UFMA), por suas orientações sobre a fitoquímica.

\section{REFERÊNCIA}

Angelo, P.M.; Jorge, N. Compostos fenólicos em alimentos: uma breve revisão. Instituto Adolfo Lutz, v. 66, n.1, p. 1-9, 2007.

Baggio, C.H.; et al. Gastroprotective mechanisms of indole alkaloids from Himatanthus lancifolius. Planta Medicinais., v. 71, n. 8, p. 733-738, 2005.

Bruneton, J. Phenols and Phenolic acids. In.: Bruneton, J. Pharmacognosy, phytochemistry and medical plants. EUA: Lavoisier Press, 1995, p. 211-227.

Colares, A.V. et al. Phytochemical and biological preliminary study of Himatanthus drasticus (Mart.) Plumel (Janaguba). Pharmacognosy Magazine, v. 4, n. 14 , p. $73-77,2008$ a.

Colares, A.V. et al Efeito gastroprotetor do látex de Himatanthus drasticus (Mart.) Plumel (Janaguba). Infarma, v. 20, n. 11/12, p. 34-36, 2008b.

Di Stasi, L.C.; Hiruma-Lima, C.A. Plantas medicinais na Amazônia e na Mata Atlântica. São Paulo: UNESP, p. 152, 2002.

França, O.O.; Brown, R.T.; Santos, C.A.M. Uleine and emethoxyaspidospermine from the bark of Plumeria lancifolia. Fitoterapia, v. 71, n. 2, p. 208-210, 2000.

Grupo, L.R.P. Contribuição ao estudo anatômico, fitoquímico e farmacológico de Synadenium carinatum Hook f. (Euphorbiaceae). 1998. $78 \mathrm{f}$. Dissertação (Mestrado em Botânica) - Universidade Federal do Paraná, Curitiba, 1998.

Honda, N.K. Estudo químico de plantas de Mato Grosso do Sul I: triagem fitoquímica. Campo Grande: EUFMS, 1990.

Ibge. Instituto Brasileiro de Geografia e Estatística. Divisão territorial do Brasil e limites territoriais.1jul.2008. Disponível em:http://pt.wikipedia.org/wiki/Caxias_ (Maranh\%C3\%A3o). Acesso em: 10 jun. 2011.

Kazmi, S. N. et al. Plumerinine - a novel lupin alkaloid from Plumeria rubra. Heterocycles, Amsterdam, v. 29, n. 10, p. 1901-1906, 1989.

Kisakurek, M.V.; Hesse, M. Chemotaxonomic Studies of the Apocynaceae. London: Academic Press, 1980.

Lorenzi, H. Árvores brasileira: manual de identificação e cultivo de plantas arbóreas nativas do Brasil. São Paulo: Plantarium, 1992.

Lorenzi, H.; Matos, F.J.A. Plantas medicinais no Brasil: nativas e exóticas: Plantarium. Nova Odessa, SP: Instituto m, 2008.

Matos, F.J.A. Plantas da medicina popular do nordeste: propriedades atribuídas e confirmadas. Fortaleza: EUFC, 1999.

Matos, F. J. A. Introdução à Fitoquímica Experimental. 2 ed. Fortaleza: Edições UFC, 1997.

Massiot, G.A. Boumendel. Alkaloids from Alstonia undulifolia. Phytochemistry, v. 31, n.3, p. 1078-1079, 1992.

Mesquita, C.T.; Xavier, S.S.; Mesquita, E.T. Insuficiência cardíaca aguda. In: CELMO, Celeno Porto (Org.). Doenças do coração: tratamento e prevenção. Rio de Janeiro: Guanabara Koogan, 2005. p. 281-284.

Miranda, G.J.; Azzam, O; Shirako, Y. Comparison of nucleotide sequences between Northern and Southern Philippine isolates of rice grassy stunt virus indicates occurrence of natural genetic reassortment. Virology., n. 266, p. 26-32, 2000.

Moreira, E.A. Marcha sistemática de análise fitoquímica. Trib.Farm., Curitiba, v. 47, n. 1, p. 1-19, 1979.

Nakashima, T. Étude phitochimique, evaluation des activités antifongiques et antivirales de trios Verbenaceae: Lippia alba N.E. Brown, Lippia multiflora Mold. Citharexylum myrianthum Cham. 1993. Tese (Doutorado) - Institut National Polytechnique de Toulouse, França, 1993.

Novaes, J. R. C.; Rapoporte, B. Espécies coletadas no Estado do Rio de Janeiro depositadas no Herbário RB. Rio de Janeiro: [s. n.], 1996.

Oliveira, R. B. Terpenos e terpenóides. 2007. Disponível em: <www.geocities.com.br/plantas tóxicas>. Acesso em: 15 ago. 2011.

Perdue, G.P.; Blomster, R. N. South American plants III: Isolation of fulvoplumierin from

Himatanthus sucuuba (M. Arg.) Woodson (Apocinaceae). Journal Pharmaceutical Sciences., v. 67, n. 9, p.13221323, 1978.

Plumel, M.M. Le genre Himatanthus (Apocinaceae): revisión taxonomique bradea. Boletim do Herbarium Bradeanu, Rio de Janeiro, v. 5, p.1-20, 1991.

Santos, R. I. Metabolismo básico e origem dos metabólitos secundários. In: Simões, C. M.

O. et al. Farmacognosia: da planta ao medicamento. 4. ed. Porto Alegre; Florianópolis: UFRGS, 2002. p. 333-365.

Schenkel, E. P. et al. Plantas tóxicas. In: SIMÕES, C. M. O. et al. (EDS.). Farmacognosia: da planta ao medicamento. 3 ed. Porto Alegre: Ed Universidade UFRGS, 2001.

Silva, J.R.A. et al. Atividade citotóxica seletiva do látex de Himatanthus sucuuba (Spruce) Woodson. In: Simpósio de Plantas Medicinais do Brasil. 15, 1998a, Águas de Lindóia. Anais... Águas de Lindóia, v. 1. p. 03117, 1998a.

Simões, C.M.O. et al. Farmacognosia: da planta ao medicamento. 6. ed. Porto Alegre: UFRG, 2007.

Sousa, F. et al. CO2 soil degassing mapping: a contribution to the volcanic risk assessment at Furnas Village (S. Miguel Island, Azores). [S. I.]: General Assembly da EGU, 2004.

Spina, A.P. Estudos taxonômicos, micro-morfológico e filogenético do gênero Himatanthus Willd. Ex Schult. (Apocynaceae: Rauvolfioideae-Plumerieae). 2004. 197f. Tese (Doutorado em Biologia Vegetal) Universidade Estadual de Campinas, Campinas, SP, 2004.

Vickery, M. L., Vickery, B. Secondary plant metabolism. Hong Kong: The Macmillan Press Ltd., 1981. 